\title{
Metastatic leiomyosarcoma of the thyroid gland: cytologic findings and differential diagnosis
}

\author{
Jiyeon Lee, Yunjoo Cho, Kyue Hee Choi, Inwoo Hwang, Young Lyun Oh \\ Department of Pathology and Translational Genomics, Samsung Medical Center, Sungkyunkwan University School of Medicine, Seoul, Korea
}

\begin{abstract}
Metastatic leiomyosarcoma to the thyroid is an extremely rare occurrence, and only 18 cases have been reported. Here, we report a case of a 37-year-old woman who presented with multiple masses on the scalp. Excisional biopsy was done and the mass revealed fascicles of smooth muscle fibers which showed positive staining for smooth muscle actin, thus confirming the diagnosis of leiomyosarcoma. The patient was also found to have a $0.9 \mathrm{~cm}$ mass within the left thyroid. Fine-needle aspiration was done and the cytological smear showed hypercellular spindle cell clusters with hyperchromatic and large nuclei. Normal thyroid follicular cells were found within or around tumor cells. In this report, we present the cytologic findings of metastatic leiomyosarcoma to the thyroid and offer differential diagnoses of the aspirated spindle cells.
\end{abstract}

Key Words: Leiomyosarcoma, secondary; Cytology; Leiomyosarcoma; Thyroid neoplasms

Received: March 25, 2021 Revised: June 14, 2021 Accepted: June 22, 2021

Corresponding Author: Young Lyun Oh, MD, PhD, Department of Pathology and Translational Genomics, Samsung Medical Center, 81 llwon-ro, Gangnam-gu, Seoul 06351, Korea Tel: +82-2-3410-2805, Fax: +82-2-3410-2831, E-mail: bijou@skku.edu

Soft tissue sarcoma metastatic to the thyroid is extremely rare, and it poses a challenge for a pathologist to diagnose it on cytology aspiration alone. Here, we report a case of metastatic leiomyosarcoma to the thyroid and describe in detail its cytologic findings.

\section{CASE REPORT}

A 37-year-old woman presented with multiple masses on the scalp. She underwent an excisional biopsy of the scalp mass at a local clinic and visited our hospital for further work up. Computed tomography of the neck revealed a poorly enhancing mass lesion in the left lobe of the thyroid measuring about 1 $\mathrm{cm}$ with ipsilateral cervical lymph node metastasis (Fig. 1A). An ultrasonographic examination of the thyroid revealed a $0.9 \mathrm{~cm}$ hypoechoic mass without increased vascularity (Fig. 1B). Ultrasound-guided fine-needle aspiration (FNA) was done to rule out the possibility of primary thyroid cancer. Unlike a typical thyroid malignancy, the aspirated material was sticky and difficult to aspirate.

The alcohol-fixed smears were stained with hematoxylin and eosin and Papanicolau for the cytologic examination. The cytological smears were hypercellular and displayed spindle cell clusters in hemorrhagic background. No necrotic material was observed. The spindle cells were arranged in a fascicular pattern and showed an indistinct cell border. The cells had eosinophilic and fibrillary cytoplasm. Individual cells showed variation in nuclear size and shape. However, the nuclei of most of the cells were hyperchromatic and large, and they demonstrated an elongated shape with blunt ends (Fig. 2A, B). Normal follicular cells were admixed with pleomorphic tumor cells, and were also observed around tumor cell clusters (Fig. 2C, D). These features raised the suspicion for a malignant tumor of mesenchymal origin.

Histological slides from the resection specimen of the scalp mass revealed a small nodular mass measuring $0.8 \times 0.6 \mathrm{~cm}$. The tumor cells were arranged in bundles or fascicles of smooth muscle fibers (Fig. 3A). They were highly pleomorphic and had a centrally located, blunted, cigar-shaped nucleus adjacent to perinuclear cytoplasmic vacuoles. The mitotic rate was high (7 mitosis per 10 high-power field), but there was no area of necrosis. Immunohistochemical staining showed a positive result for smooth muscle actin (SMA) and desmin (Fig. 3). 

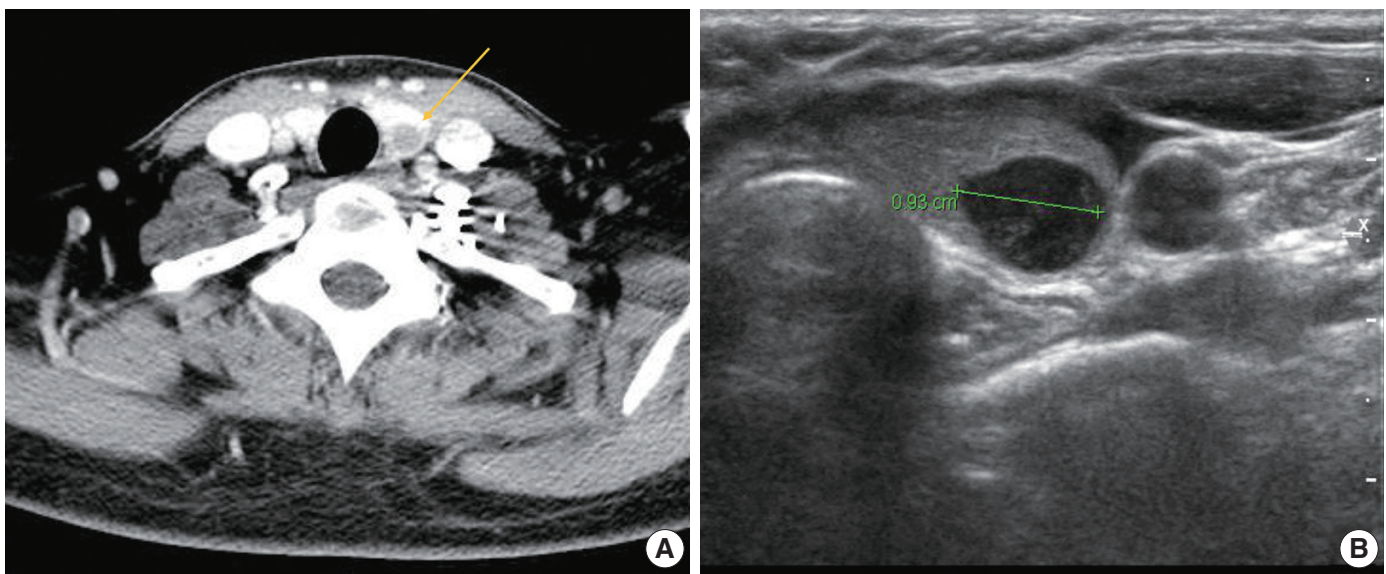

Fig. 1. Radiologic findings of metastatic leiomyosarcoma. (A) Computed tomography scan reveals $1 \mathrm{~cm}$ sized poorly enhancing space occupying lesion (arrow) in left thyroid. (B) Ultrasound shows marked hypoechoic nodule in the inferior pole of left thyroid.
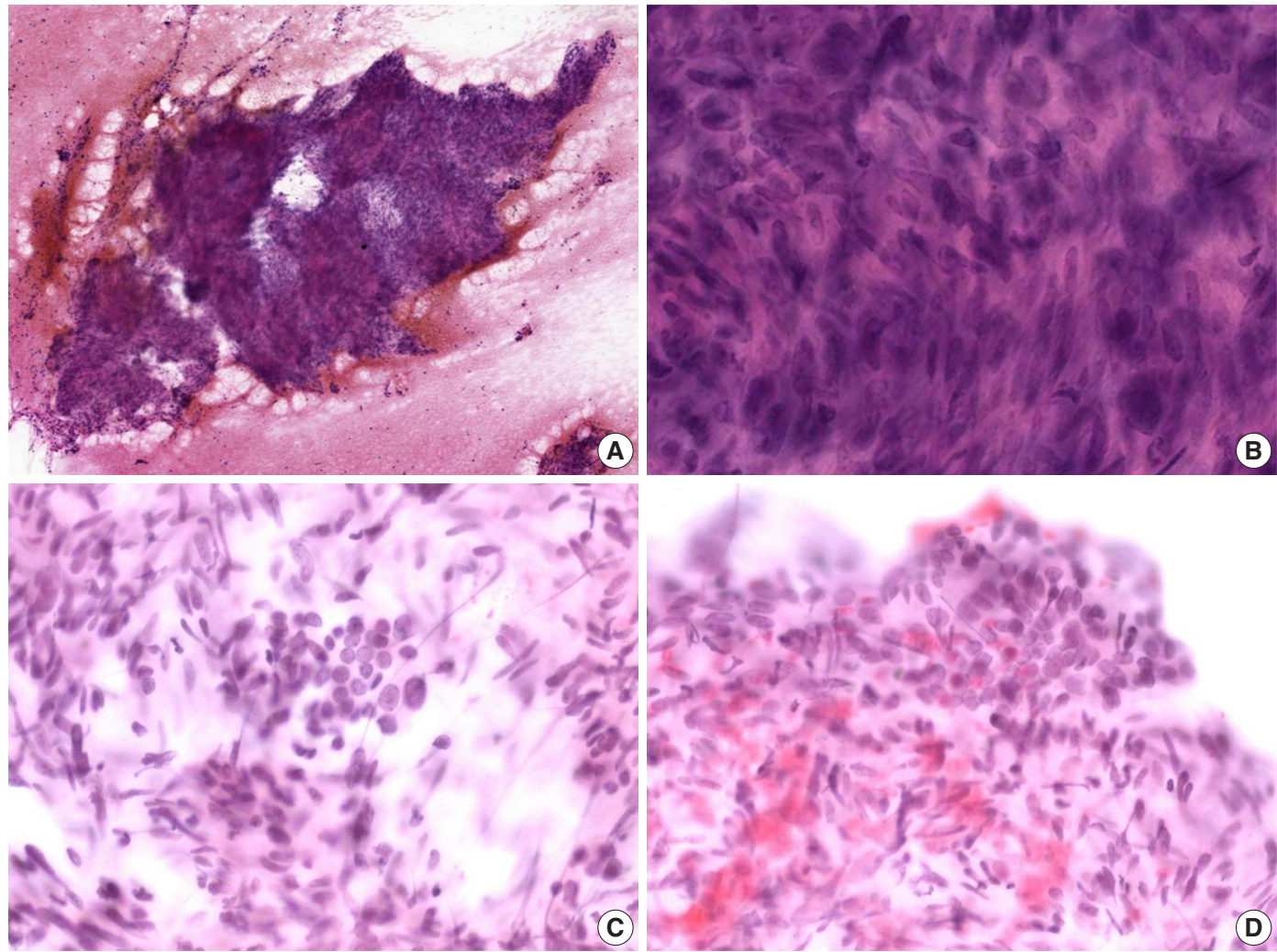

Fig. 2. Cytologic features of metastatic leiomyosarcoma. (A) Hypercellular spindle cell clusters are arranged in a fascicular pattern. (B) The cells show indistinct cell borders, eosinophilic cytoplasm, and hyperchromatic, large, elongated nuclei with blunt ends. (C, D) Normal follicular cells are admixed with pleomorphic tumor cells (C) or discovered around tumor cell clusters (D).

Positron emission tomography-computed tomography revealed several hypermetabolic masses on the right side of the abdomen, the largest of which measured $5.8 \mathrm{~cm}$. One mass was located in the mesentery, which had no association with the uterus. In addition, there were multiple hypermetabolic lesions in the left internal mammary area, both thigh, and the lung. To exclude other spindle cell tumors which can arise in the abdominal cavity, immunostaining for S100, CD34, MDM2, c-Kit, and anaplastic lymphoma kinase were performed. All of the additional immunohistochemical stainings turned out negative, and the possibility of schwannoma, solitary fibrous tumor, liposarcoma, gastrointestinal stromal tumor and inflammatory myofibroblastic 

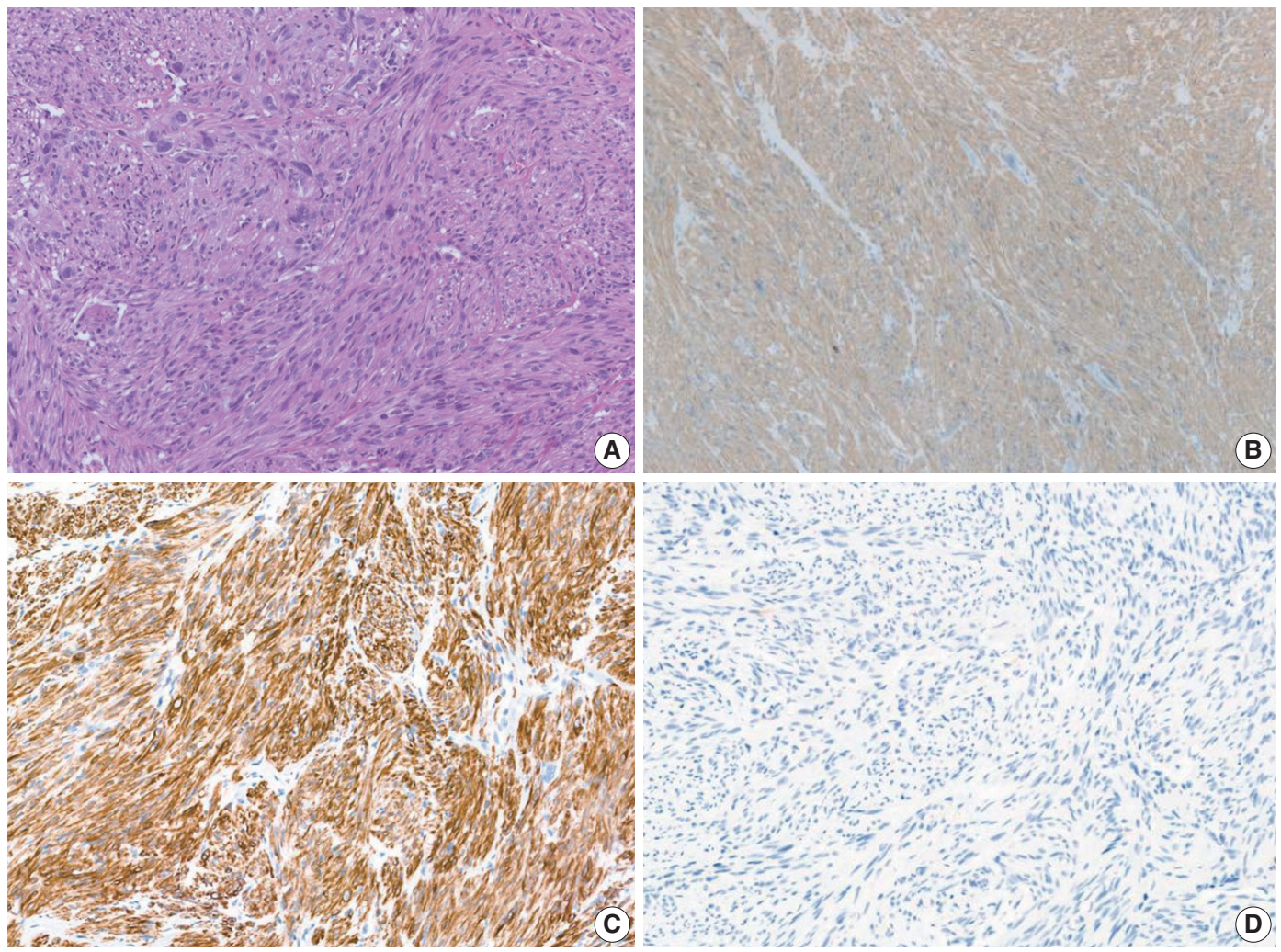

Fig. 3. Histologic features of the scalp mass. (A) Excisional biopsy specimen from the scalp mass shows bundles or fascicles of smooth muscle fibers. The tumor cells are highly pleomorphic and have a centrally located, blunted, cigar-shaped nucleus adjacent to perinuclear cytoplasmic vacuoles. (B, C) Smooth muscle actin (B) and desmin (C) immunostaining shows positivity. (D) c-Kit immunostaining shows negativity.

tumor were ruled out, respectively. Considering all the clinical, morphological and immunohistochemical features, we determined that the final diagnosis of the tumor located in the thyroid gland to be metastatic leiomyosarcoma from the mesentery. Because of the presence of metastatic disease, the patient received doxorubicin-based chemotherapy instead of further surgical intervention. She is still alive 22 months after her first visit.

\section{DISCUSSION}

Metastasis of sarcoma to the thyroid is rare with a few cases reported in the literature. To date, 18 cases have been reported as metastatic leiomyosarcoma to the thyroid, most of which are from uterine leiomyosarcoma (Table 1). Furthermore, it is very rare to be diagnosed using FNA cytology [1-3].

FNA cytology of metastatic leiomyosarcoma tends to show hypercellular spindle cell clusters with marked pleomorphism, and there can be conspicuous mitoses. Therefore, the differential diagnosis should include primary thyroid tumors showing spindle cell features; such as medullary carcinoma, anaplastic carcinoma and spindle epithelial tumor with thymus-like differenti- ation (SETTLE). Likewise, primary or metastatic mesenchymal tumors like malignant peripheral nerve sheath tumor (MPNST) or synovial sarcoma should be considered.

Medullary thyroid carcinoma is one of the first differential diagnosis to consider when spindle cells are observed in thyroid FNA. However, the characteristic cytology of medullary carcinoma is dispersed single cells having "salt-and-pepper" nuclei. In most cases, oval-to-plasmacytoid cells were observed together.

Cytomorphologically, SETTLE has a close resemblance to leiomyosarcoma. They can be shown as variably sized groups of spindle cells with uniform, elongated, and cigar-shaped nuclei similar to those of leiomyosarcoma [4]. In addition, epithelial components of SETTLE can mimic benign follicular cells that appear in metastatic leiomyosarcoma. However, diffuse immunoreactivity for cytokeratin in both components is the most obvious difference from leiomyosarcoma.

Anaplastic carcinomas also have spindle-shaped cells. However, anaplastic carcinomas rarely occur below the age of 50 and usually present as a rapidly growing mass infiltrating surrounding soft tissues. Spindle cells in anaplastic carcinoma show a more bizarre morphology compared to that shown in leiomyosarcoma 


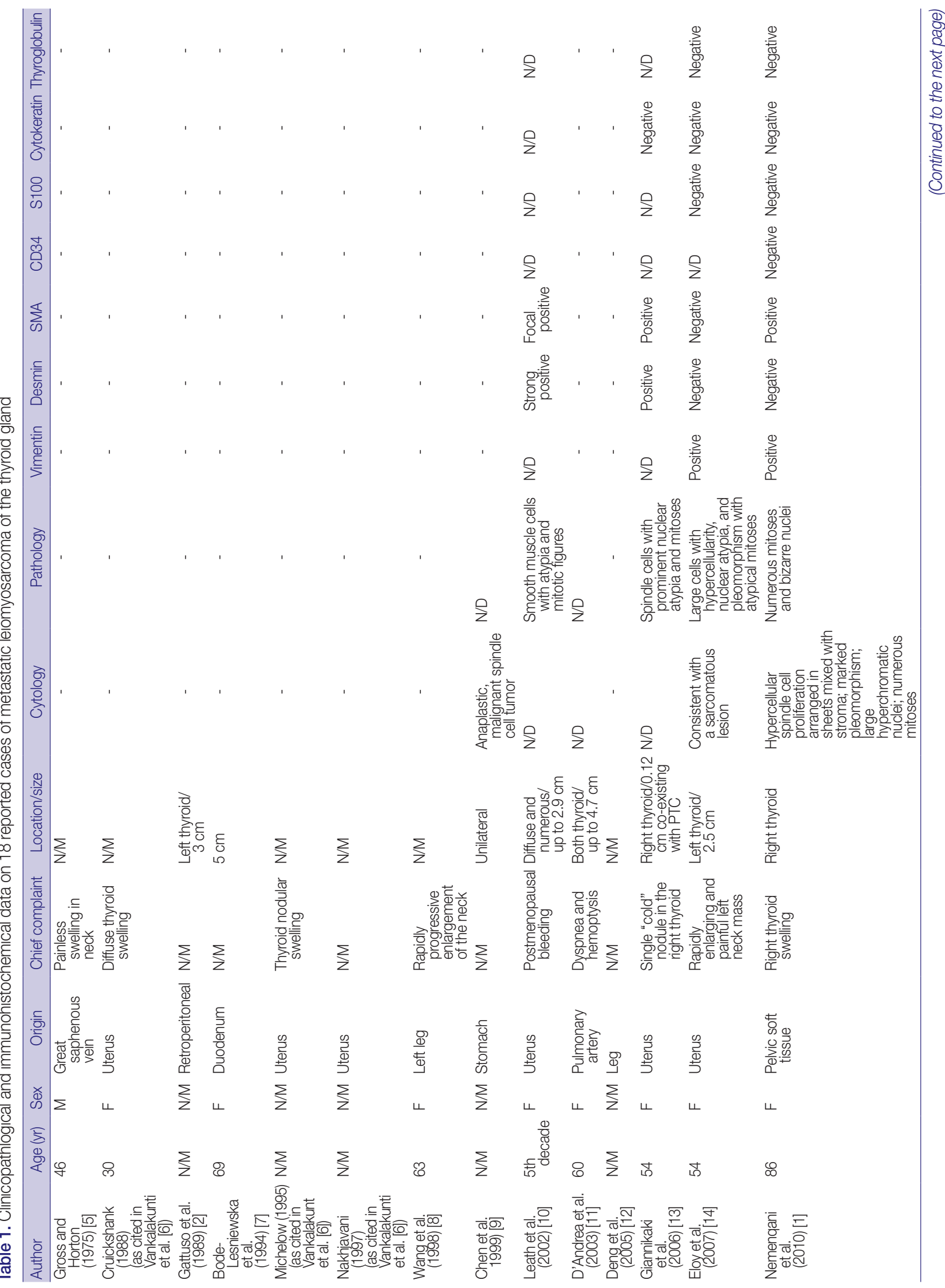




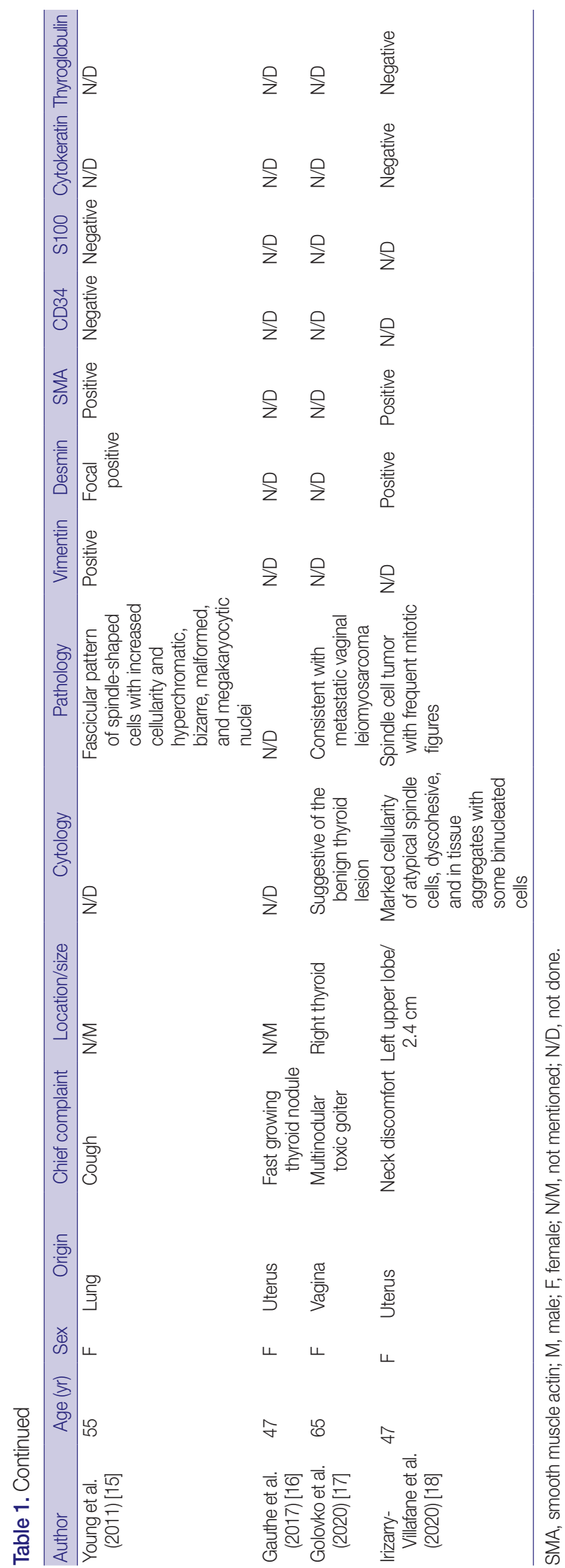

[19]. Osteoclast-like giant cells, necrosis, and mitotic figures are a common feature, and in more than half of the case, anaplastic carcinomas are positive for cytokeratin. PAX8 is positive in about $80 \%$ of anaplastic carcinomas.

MPNST can present as highly cellular clusters of spindle cells showing wavy, elongated nuclei with tapered ends [20]. The cytologic features of synovial sarcoma include hypercellular and uniform oval-to-spindle cells without nuclear pleomorphism or necrosis. Despite its biphasic nature, the epithelial component is rarely observed in the FNA specimen.

Although there are differences between the differential diagnoses as described above, it is nearly impossible to conclusively diagnose leiomyosarcoma in FNA slides with only histologic observation of the cells in cytologic smears. Immunohistochemistry is crucial for a precise diagnosis. Leiomyosarcoma is positive for vimentin, SMA, muscle-specific actin, desmin and h-caldesmon and negative for cytokeratin, thyroglobulin, calcitonin, and S-100 protein. In our case, we diagnosed leiomyosarcoma by confirming that the resected scalp mass was positive for SMA and desmin and negative for S-100 protein, CD34, MDM2, c-Kit, and anaplastic lymphoma kinase. It is difficult to make a distinction between primary and metastatic leiomyosarcoma cytologically. Metastases to the thyroid gland occur more frequently than is generally appreciated and can be easily misdiagnosed as a primary thyroid tumor. Given the rarity of thyroid primary sarcoma, pathologists should be alert to the possibility of metastatic tumor, especially when benign follicular cells are identified within or around malignant cells.

\section{Ethics Statement}

This study was approved by the Institutional Review Board of Samsung Medical Center with a waiver of informed consent (IRB No. 2021-03-125).

\section{Availability of Data and Material}

The datasets generated or analyzed during the study are available from the corresponding author on reasonable request.

\section{Code Availability}

Not applicable.

\section{ORCID}

Jiyeon Lee Yunjoo Cho Kyue Hee Choi Inwoo Hwang Young Lyun Oh

\author{
https://orcid.org/0000-0002-4457-7499 \\ https://orcid.org/0000-0003-1309-2015 \\ https://orcid.org/0000-0001-8386-5349 \\ https://orcid.org/0000-0002-7749-3929 \\ https://orcid.org/0000-0002-9127-4642
}

\section{Author Contributions}

Conceptualization: YLO. Data curation: JL. Formal analysis: JL. Investigation: JL, YC, IH, KHC, YLO. Methodology: JL, YC. Resources: YLO. Super- 
vision: YLO. Validation: YLO, JL. Visualization: JL, YC. Writing—original draft: JL. Writing-review \& editing: YLO, YC. Approval of final manuscript: all authors.

\section{Conflicts of Interest}

The authors declare that they have no potential conflicts of interest.

\section{Funding Statement}

No funding to declare.

\section{References}

1. Nemenqani D, Yaqoob N, Khoja H. Leiomyosarcoma metastatic to the thyroid diagnosed by fine needle aspiration cytology. J Pak Med Assoc 2010; 60: 307-9.

2. Gattuso P, Castelli MJ, Reyes CV. Fine needle aspiration cytology of metastatic sarcoma involving the thyroid. South Med J 1989; 82: 1158-60.

3. Michelow PM, Leiman G. Metastases to the thyroid gland: diagnosis by aspiration cytology. Diagn Cytopathol 1995; 13: 209-13.

4. Tong GX, Hamele-Bena D, Wei XJ, O'Toole K. Fine-needle aspiration biopsy of monophasic variant of spindle epithelial tumor with thymus-like differentiation of the thyroid: report of one case and review of the literature. Diagn Cytopathol 2007; 35: 113-9.

5. Gross E, Horton MA. Leiomyosarcoma of the saphenous vein. J Pathol 1975; 116: 37-41.

6. Vankalakunti M, Kaur J, Srinivasan R. Cytology of sarcoma metastasizing to the thyroid: a case report. Acta Cytol 2008; 52: 729-32.

7. Bode-Lesniewska B, Schroder S, Gemsenjager E, Staubli M, Pfaltz M. Leiomyosarcoma in the thyroid gland: primary tumor or metastasis?. Pathologe 1994; 15: 303-7.

8. Wang TY, Lee CH, Yang AH, P’Eng FK, Lui WY. Metastatic leiomyosarcoma of the thyroid: a case report. Zhonghua Yi Xue Za Zhi (Taipei) 1998; 61: 353-7.

9. Chen H, Nicol TL, Udelsman R. Clinically significant, isolated met- astatic disease to the thyroid gland. World J Surg 1999; 23: 177-80.

10. Leath CA 3rd, Huh WK, Straughn JM Jr, Conner MG. Uterine leiomyosarcoma metastatic to the thyroid. Obstet Gynecol 2002; 100: $1122-4$.

11. D'Andrea N, Romano V, Mattioli F, et al. Pulmonary artery leiomyosarcoma with thyroid metastases. Monaldi Arch Chest Dis 2003; 59: 304-7.

12. Deng XR, Wang G, Kuang CJ, Peng GZ, Chen RS. Metastasis of leiomyosarcoma to the thyroid. Chin Med J (Engl) 2005; 118: 174-6.

13. Giannikaki E, Mantadakis E, Mamalaki E, Delides G, Samonis G. Metastatic uterine leiomyosarcoma coexisting with papillary carcinoma of the thyroid gland. Int J Gynecol Cancer 2006; 16: 442-5.

14. Eloy JA, Mortensen M, Gupta S, Lewis MS, Brett EM, Genden EM. Metastasis of uterine leiomyosarcoma to the thyroid gland: case report and review of the literature. Thyroid 2007; 17: 1295-7.

15. Woo Young K, Young Ran K, Sang Uk W, Jae Bok L. Pulmonary leiomyosarcoma metastatic to the thyroid gland: case report and review of the literature. Ann Endocrinol (Paris) 2011; 72: 314-6.

16. Gauthe M, Testart Dardel N, Nascimento C, Trassard M, Banal A, Alberini JL. Uterine leiomyosarcoma metastatic to thyroid shown by (18)F-FDG PET/CT imaging. Rev Esp Med Nucl Imagen Mol 2017; 36: 113-5.

17. Golovko A, Kuryk O, Tkachenko R, Mendel N. Thyroid metastasis of vaginal leiomyosarcoma: a case report and review of the literature. Exp Oncol 2020; 42: 242-4.

18. Irizarry-Villafane G, Rivera-Santana N, Mangual-Garcia M, et al. A rare case of uterine leiomyosarcoma with metastasis to the thyroid gland. Case Rep Endocrinol 2020; 2020: 8889843.

19. Papi G, Corrado S, LiVolsi VA. Primary spindle cell lesions of the thyroid gland: an overview. Am J Clin Pathol 2006; 125 Suppl: S95123.

20. Sanchez B, Dutra J, Nayar R, Lin X. Metastatic malignant peripheral nerve sheath tumor to the thyroid. J Thyroid Cancer 2015; 1: 18-23. 